\title{
A metagene of NRF2 expression is a prognostic biomarker in all stage colorectal cancer
}

Séan M. O’Cathail ${ }^{1 *} \square$, Chieh-Hsi Wu ${ }^{2} \square$, Annabelle Lewis ${ }^{3}$, Chris Holmes ${ }^{2,4,5}$, Maria A

Hawkins $^{1}$, Tim Maughan ${ }^{1}$

1 Oxford Institute of Radiation Oncology, University of Oxford, UK

2 Department of Statistics, University of Oxford, OX1 3LB, UK

3 Wellcome Trust Centre for Human Genetics, University of Oxford, UK

4 Nuffield Department of Medicine, University of Oxford, UK

5 Alan Turing Institute, London, UK

* Corresponding author

$\square$ Denotes equal contribution

Keywords: NRF2, colorectal cancer, metagene, biostatistics

Conflicts of interest: Dr. Maughan reports grants from Medical Research Council, grants from Cancer Research UK, during the conduct of the study; non-financial support and other from Astra Zeneca, personal fees from Pierre Fabre, outside the submitted work. Dr Holmes reports funding from Novartis for research collaboration outside the submitted work.

Word count: 3258 


\section{ABSTRACT}

\section{Objective}

Nrf2 overexpression confers poor prognosis in some cancers but its role in colorectal cancer

(CRC) is unknown. Due to its role as a transcription factor we hypothesise a metagene of

NRF2 regulated genes could act as a prognostic biomarker in CRC.

\section{Design}

Using known NRF2 regulated genes, we defined an NRF2 metagene to represent the pathway expression using principal component analysis and Cox proportional hazard models. The NRF2 metagene was validated in four independent datasets, including the recently profiled MRC FOCUS randomised controlled trial.

\section{Results}

36 genes comprised the final prognostic metagene in the training set. 1,360 patients were included in the validation analyses. High NRF2 metagene expression is associated with worse disease free survival (DFS) and overall survival (OS) outcomes in stage I/II/III disease and worse OS in stage IV disease. In multivariate analyses, NRF2 expression remained significant when adjusted for known prognostic factors of adjuvant chemotherapy and stage in stage I/II/III disease, as well as BRAF V600E mutation and sidedness in stage IV disease. NRF2 metagene expression exhibits variation within each of the Consensus Molecular Subtypes (CMS) but high expression is particularly enriched in CMS 4.

\section{Conclusion}

We demonstrate in a large scale analysis that NRF2 expression is a novel biomarker of poor prognosis across all stages of colorectal cancer. Higher expression observed in CMS 4 further refines the molecular taxonomy of colorectal cancer. 


\section{INTRODUCTION}

Colorectal cancer is the $4^{\text {th }}$ most common cancer in the UK with 41,700 new cases per annum and only $57 \%$ of patients will live ten years or more [1]. An improved understanding of the biology of colorectal cancer may provide the basis for stratification of patients for differing treatment programmes, first by identifying prognostic effects. Current, known prognostic factors include 'sidedness' (left or right side of the colon) [2] — which is assumed to be surrogate of tumour [3] and patient biology [4]—RAS mutant status, BRAF status [5] and mismatch repair status [6,7]. The most widely used, RNA expression based classifier is the Consensus Molecular Subtype (CMS) [8]. It highlights that colorectal cancer is a significantly heterogeneous disease with different prognostic expectations among four subgroups. The taxonomy applied to each of the four CMS subtypes indicate enriched pathways, with no subtype defined by individual events, genetic aberrations or expression pathways.

The KEAP1-NRF2 pathway is a canonical stress response pathway which has shown prognostic importance in many tumours types, notably lung cancer [9]. NRF2 is a potent transcriptional activator that plays a central role in cell protection against oxidative and electrophilic stress. NRF2 activity is tightly regulated by KEAP1. Under basal (unstressed) conditions KEAP1, part of the Cul3 ubiquitin ligase family, mediates polyubiquitination of NRF2 protein [10]. During cellular stress changes in the thiol rich protein, KEAP1 modifies the structural integrity of the KEAP1-CUL3 ligase complex resulting in declining ubiquitination activity and an increase in cellular NRF2. NRF2 translocates to the nucleus and binds to antioxidant response element (ARE) sequences to regulate the transcription of suites of genes, including intracellular redox control, metabolic pathways, autophagy and drug transport [11]. Historically the NRF2 pathway was deemed to function in 'tumour 
suppressor' like capacity, allowing the cell to defend against stressors such as carcinogens [11]. However more recent evidence shows that some tumours acquire constitutive activation of the pathway which allow it to function in an 'oncogene' like fashion, promoting cell survival, resisting radiation, chemotherapeutics and dysregulating metabolism [12,13].

There are a number of distinct mechanisms by which the NRF2 pathway can become constitutively activated [14]. Mutations in both KEAP1 and NFE2L2 have been described in a up to $7.8 \%$ of colorectal cancers [15], although the rate in TCGA was less than $2.4 \%$ and $0.9 \%$ respectively [16]. Epigenetic modifications, methylation, of KEAP1 in colorectal tumours can silence its ability to regulate NRF2 [17]. Direct activation of NRF2 transcription via activated oncogenes such as $K R A S^{G 12 D}, B R A F^{V 619 E}$ and $c-M Y C^{E R T 2}$ has also been described [18]. Indeed in CRC, the level of NRF2 signalling in the TCGA datasets is higher than would expect for the low somatic mutation rate observed [19], further suggesting complex post transcriptional mechanisms of activation.

Therefore it seems unlikely that NFE2L2 expression in isolation will capture the full effect of pathway expression, and prove a useful biomarker. However, as NRF2 functions as a transcription factor controlling a battery of antioxidant response element (ARE) regulated genes, we hypothesise that a 'metagene' of NRF2 expression could be used to aggregate different mechanisms of pathway expression and act as a biomarker of prognosis in colorectal cancer. Here we detail for the first time the derivation of an NRF2 metagene from RNA expression data using a candidate gene approach [20] in colorectal cancer and demonstrate that high NRF2 expression is an independent biomarker of poor prognosis across all CRC disease stages. 


\section{METHODS}

\section{Candidate gene selection}

Known NRF2 regulated genes were selected from two published prognostic lung cancer signatures [21,22] and refined for differential expression using the Oncomine database [23]. Input parameters "Cancer Type" and "Analysis Type" were set to "colorectal cancer and 'cancer versus normal' respectively. Differential expression was determined by threshold values of fold change $>2$, p-value $<0.0001$ and gene rank of top $10 \%$. The database normalised gene expression across all selected datasets to allow summative gene expression comparisons. The resulting median gene rank for the meta-analysis across all selected datasets was calculated with its associated p-value, which was corrected for multiple hypothesis testing using the false discovery rate method [24].

\section{Construction of the NRF2 metagene}

Principal component analysis (PCA) was applied to the probes representing the candidate NRF2 target genes. This generated a new set of continuous variables, principal components (PCs), which were weighted averages of the RNA expressions across the probes considered. Supervised variable selection was performed to decide how many major $\mathrm{PCs}^{1}$ would be useful for predicting prognosis in the training set. A Cox proportional hazard regression model was used to model the prognosis predicted by the PCs of NRF2 expression.

The NRF2 metagene in each validation set was obtained by performing PCA on the corresponding probe sets (see supplementary figure 1 and supplementary information for further details).

\footnotetext{
${ }^{1}$ Here, we define the X major PCs as the X most variable PCs that explains $80 \%$ of the variation in the data.
} 


\section{Datasets}

Publically available colorectal datasets were downloaded from the Gene Expression Omnibus (GEO) database. Clinical and expression data was accessed from R programming environment using the packages 'GEOquery' [25] and 'Biobase' [26] obtained from https://bioconductor.org/biocLite.R. All datasets are summarised in Table 1. The datasets were divided into a training set, GSE17536 [27] and validation sets. Validation was carried out using datasets representative of non-metastatic, stage I-III disease (GSE14333 and GSE39582) [28,29], metastatic disease (MRC FOCUS trial) [30] and rectal only cancer (GSE87211) [31]. As part of the MRC Stratification in Colorectal cancer (S:CORT) consortium, we had access to the MRC FOCUS trial data including the RNA expression profiles generated by S:CORT (See supplementary information).

\section{Statistical analysis}

\section{Primary analysis}

The primary analysis for each validation set was to test whether the NRF2 metagene has a prognostic effect on disease free survival (DFS) and/or overall survival (OS). A Cox PH regression model with no variable was constructed, representing the null model. A second Cox PH regression model is fitted with the NRF2 metagene. The likelihood ratio test (LRT) is used to provide evidence against the null hypothesis (H0), that the NRF2 metagene provides no explanatory power. For the construction of Kaplan-Meier (K-M) curves, NRF2 metagene expression was subdivided by tertiles. Hazard ratios, and confidence intervals, presented for these curves are between the upper and lower tertiles. Significance testing was done only on Cox model analysis.

\section{Secondary analyses}

To assess whether the prognostic effect of the NRF2 metagene was confounded with other known prognostic variables we performed adjusted analyses using multivariate Cox PH 
regression models. Because the adjusting variables available varied across datasets, no adjusting variables were used in the training set. Prior to the adjusted analysis, the $\mathrm{R}$ package MICE [32] was used to impute missing values in the adjusted variables in GSE39582 and MRC FOCUS trial data. A LRT was performed to test the H0 that the model with only the adjusting variables adequately approximates the model with the adjusting variables and the NRF2 metagene. Significance suggests that NRF2 expression provides additional information to explain the survival outcome. The adjusting variables used for the secondary analyses are summarised in Table 1. All statistical analyses were conducted using the statistical computing software R [33].

\section{RESULTS}

\section{NRF2 metagene derivation and training}

In total 62 candidate genes were analysed in a total of 9 independent colorectal datasets for differential expression relative to normal tissue [16,34-39]. Some datasets were subsetted into different anatomical sites for the purposes of analysis resulting in 24 discrete sets of data (see supplementary Table 1). 40 were found to be differentially expressed, 21 of which were significantly over-expressed and 20 which were significantly under-expressed, in at least one or more of the datasets (Figure 1A). One gene, COL3A1, was shared as it was over expressed in some datasets and under expressed in others. Of the 40 differentially expressed genes, four could not be matched between the training and validation datasets so were omitted from further analysis. The final group of 36 genes was: $A B C A 8, A B I 3 B P, A D A M 12, A D R B 1$, ANGPT1, ANKRD29, ANKRD44, BCHE, C15orf48, COL3A1, COL5A1, EGLN3, LIFR, METTL7A, PCM1, PLAU, PLCB4, RECK, RGCC, RRM2, SEC14LA, SERPINH1, SFN, SLIT3, SPP1, TNS1, TOM1L2, TSPAN5, TTYH3, VSIG10, VCAN, AKR1C1, LRP8, NAMPT, PTGES, SLC27A5. There was a very high level of co-ordinated expression between the 
majority of the 36 genes in the training dataset as evidenced by pairwise correlations (Figure 1B). This group of genes is designated the NRF2 metagene.

\section{Principal Component Analysis}

In the training set the 36 genes are represented by 92 probes, where 65 of the 92 probes have an absolute correlation (in expression) $>0.5$ with another probe. This indicated potentially strong dependencies among the probes and so the expression levels measured by the 92 probes could be represented by a smaller number of variables. The 11 major PCs explained up to $80 \%$ of the variation in the 92 probes. The strong dependency among the probes was confirmed by the fact that the 81 minor PCs only explained $<20 \%$ of the remaining variation.

\section{Variable selection}

AIC indicated that only the two major PCs, PC1 and PC2, were useful for predicting survival outcome, while BIC suggested that only the most variable PC (PC1) was useful. The inclusion of PC2 only slightly improved AIC, we therefore only considered PC1 in the training set. $\mathrm{PC} 1$ in the training set had absolute correlations $>0.5$ with probes that mapped to the following 10 genes: VCAN, ADAM12, COL3A1, COL5A1, SERPINH1, RECK, PLAU, SPPI, TNS1 and SLIT3. Due to the high correlation of these genes with PC1 in the training set, we hypothesised that they were of higher biological relevance for prognosis prediction than other NRF2 target genes.

\section{NRF2 metagene a biomarker of worse survival}

In stage $\mathrm{I} / \mathrm{II} / \mathrm{III}$ disease, higher NRF2 metagene expression corresponded to worse DFS in

GSE14333 $\left(\mathrm{HR}^{2}=1.551,95 \%\right.$ C.I 1.200-2.004; LRT, $\left.\mathrm{p}=0.0008\right)$ and GSE39582

$(\mathrm{HR}=1.172,95 \%$ CI 1.008-1.362; LRT, $\mathrm{p}=0.0383)$. Including the 60 cases of stage IV

\footnotetext{
${ }^{2}$ As the NRF2 metagene expression is a continuous variable, the HR reported in the text of this section is the HR between the upper and lower tertiles of the NRF2 metagene expression.
} 
disease also available in GSE39582, NRF2 expression was also associated with worse OS $(\mathrm{HR}=1.240,95 \%$ C.I 1.086-1.416; LRT, $\mathrm{p}=0.001)$. In the MRC FOCUS trial, comprised of first line stage IV metastatic patients, NRF2 expression was again associated with a worse overall survival $(\mathrm{HR}=1.140,95 \%$ C.I $1.035-1.255 ; \mathrm{LRT}, \mathrm{p}=0.008)$. Figure 2 shows that high expression corresponded with worse prognosis for DFS in GSE14333 and GSE39582 (panels A and B), and for OS in GSE39582 and MRC FOCUS trial (panels C and D). In order to assess the relevance of NRF2 expression in rectal cancer specifically, and the ability to migrate between RNA expression platforms, we performed the analysis on a rectal cancer only expression dataset, where all sampled patients received neoadjuvant chemoradiotherapy (GSE87211). Higher expression was associated with worse DFS $(\mathrm{HR}=1.431,95 \%$ C.I 1.060-1.933; LRT, $\mathrm{p}=0.056)$ but not OS $(\mathrm{HR}=1.464,95 \%$ C.I $0.955-$ 2.245; LRT, $\mathrm{p}=0.197)$. Figure 3 shows that high expression corresponded to worse prognosis for DFS.

\section{NRF2 metagene provides additional explanatory power to known}

\section{prognostic variables}

Within the publically available datasets there were additional variables that are known prognostic factors. These magnitude of their respective effects are summarised in the forest plot (supplementary figure 2). We used these known prognostic factors in a multivariate analysis. In GSE14333, after adjusting for the effect of stage and adjuvant chemotherapy NRF2 expression remained a significant predictor of worse DFS $\left(\mathrm{HR}^{3}=1.365,95 \%\right.$ C.I 1.049-1.776; LRT, $p=0.02$ ). Similarly in GSE39582, the effect of high NRF2 expression corresponds to worse DFS (HR=1.168, 95\% C.I 1.000-1.363; LRT, p = 0.049) after adjusting

\footnotetext{
${ }^{3}$ See footnote 2 .
} 
for the effect of stage and mismatch repair status (MMR). In the latter dataset, NRF2 expression was also significantly associated with worse OS when adjusting for stage alone $(\mathrm{HR}=1.185,95 \%$ C.I 1.040-1.350; LRT, $\mathrm{p}=0.01)$. No adjusted analysis was carried out for MMR with NRF2 expression on OS due to the known contrasting effects MMR status has on prognosis in early stage and metastatic disease, which could lead to model misspecification. In the MRC FOCUS trial, prognostic factors within the dataset were site of the primary tumour (sidedness) and $B R A F^{\mathrm{V} 600 \mathrm{E}}$ mutation and used as adjusting variables in a multivariate analysis. Again, high NRF2 expression corresponds to worse overall survival (HR=1.123, 95\% C.I 1.020-1.237; LRT, $\mathrm{p}=0.0185)$. In summary, there was systematic evidence that NRF2 had an effect on DFS and/or OS in all available datasets (Table 2).

\section{NRF2 expression and Consensus Molecular Subtypes (CMS)}

In order to understand how NRF2 expression aligns with the current transcriptomic landscape of colorectal cancer, we examined the distribution of the three groups of NRF2 metagene expression level across the four CMS subtypes in the MRC FOCUS trial (Figure 4). While high NRF2 expression can be seen across all subtypes, strikingly CMS 4 showed substantially higher NRF2 expression with no patients in the category of low NRF2 expression. By contrast, the majority of patients in CMS 2 or 3 had low and intermediate NRF2 metagene expression.

\section{DISCUSSION}

In spite of the accumulating evidence that NRF2 plays a significant role in cancer $[40,41]$ there is remarkably little data on the prognostic contribution of NRF2 in colorectal cancer. High levels of NRF2 expression within resected tumours have been found to be significantly correlated with p53 expression, Duke's stage and poor clinical outcomes [42] as well as 
tumour size, TNM stage and metastases [43]. Whilst the latter investigated the association between NRF2 and survival status, their analysis did not take into account of the various aspects of time-to-event data, including data censoring or the concept of time. In addition, it was impossible to directly measure the prognostic effect of NRF2 in presence of potential confounders in their analysis framework. Therefore we set out to understand the effect of NRF2 expression in CRC and whether it provided additional information on prognosis to other established clinical and molecular variables.

We derived an NRF2 metagene to measure activation of the pathway in colorectal cancer and independently validated it as a biomarker of poor prognosis across all stages of colorectal cancer for the first time. This is an entirely new biological insight into colorectal cancer for several reasons.

To the authors' knowledge, this is the first demonstration of NRF2 regulated genes behaving in a co-ordinated network fashion in CRC, in vivo, and supports our 'metagene' approach to represent KEAP1/NRF2 pathway expression. The high degree of co-expression seen between key genes VCAN, ADAM12, SPP1, COL3A1, COL5A1, TNS1, SLIT3, RECK, PLAU and SERPINH1 was consistent with the predicted behaviour of these genes on the STRING database [44] (http://string-db.org/; supplementary figure 3). That the candidate genes were chosen on the basis of NRF2 regulation in lung cancer strengthens the unbiased nature of the analysis. With the exception of COL3A1, all of the key genes contain ARE within their promoter region emphasising NRF2's ability to directly regulate transcription (supplementary information, supplementary figure 4).

Secondly, the NRF2 metagene has been shown as a biomarker of poor prognosis across all stages of colorectal cancer in several large, independent datasets comprising 1,360 patients making it one of the largest validation analyses of a transcriptomic biomarker in CRC to date. 
To place the analysis size in context, the OS and DFS cohorts used to validate CMS were 2,129 and 1,785 cases respectively [8].

Thirdly, the effect was detected from three different expression platforms using both FFPE and fresh frozen tissue. Lastly, and arguably most importantly, the prognostic effect is maintained when adjusting for known prognostic clinical and molecular factors including stage, adjuvant chemotherapy and mismatch repair status in the non-metastatic setting, and furthermore, $B R A F^{V 600 E}$ mutation and tumour sidedness in the relapsed setting. The 375 patients with stage IV metastatic disease have been selected from a large randomised controlled phase III trial, which makes the findings highly stable and robust.

CMS has defined the current molecular taxonomy of CRC and is one of the most widely used RNA expression based biomarkers in CRC. The resulting classifications (CMS 1-4) and their respective prognostic outlooks describe the landscape in which any novel expression biomarker must be evaluated, especially as CMS was derived using a network-based clustering approach, agnostic of underlying biological mechanisms. We have shown that, although distributed across all four subtypes, high NRF2 expression is significantly enriched in CMS 4. This was unexpected. A priori, given that NRF2 was primarily known as a metabolic pathway and the literature supporting oncogenic KRAS activation of the NRF2 pathway, one could have expected enrichment within CMS 3, not CMS 4. High levels of NRF2 activation can promote angiogenesis and epithelial mesenchymal transition [41]. We would argue therefore that this adds further biological insight into the transcriptomic taxonomy of the CMS classification. The exact role of NRF2 in interacting with the current subtype annotations CMS 4 warrants further study.

The difference in prognosis between those with high and low NRF2 expression could, at least in part, be due to therapeutic resistance. Fluorouracil is the main chemotherapy drug used in 
both the adjuvant and metastatic setting, and was the backbone of therapy used in the FOCUS trial. Silencing of NRF2 signalling has been shown to overcome 5-FU resistance in colorectal cancer models in both an in vitro and in vivo setting [45]. Quantifying the effect of NRF2 expression in therapeutic resistance in relation to radiation and chemotherapy in colorectal cancer is ongoing in our laboratory. However, given the pluripotent nature of NRF2 it would seem plausible that it mediates poor prognosis by influencing multiple mechanisms [41].

Some limitations should be addressed. The amount of rectal tumours was low in the analysed data and the effect appears statistically less marked in the rectal only dataset GSE87211. However any argument that the finding is only pertinent to colon cancer ignores the fact that non-hypermutated colon cancers and rectal cancers are not distinguishable at the genomic level [16]. In fact, anatomical left colon more closely resembles rectum than right colon from a molecular biology standpoint. Furthermore, having accounted for sidedness and MSI where available, high NRF2 pathway expression remains a feature of poor prognosis. The statistical variance seen in GSE87211 is more likely the result of the relatively smaller number of events occurred (22\% and $13 \%$ for DFS and OS respectively) which mitigated power. It may also due to an artefact of migrating between RNA expression platforms rather than a change in biological effect between colon and rectum.

Although we used a 36 gene 'metagene' to represent NRF2 pathway expression, NRF2 is known to regulate a large number of gene targets [46]. There may be an alternative group NRF2 targets which could better represent pathway expression in colorectal cancer.

Fundamentally, the purpose of our analysis was representing NRF2 pathway expression from transcriptomic data to assess and understand its biological relevance in colorectal cancer. 
This is the first rigorous demonstration of its association with worse clinical outcomes in CRC.

The NRF2 metagene is a novel expression based prognostic biomarker, where higher expression associates with poorer prognosis across all stages of colorectal cancer. While the KEAP1-NRF2 pathway is increasingly well understood, better characterisation of its role and relationship to other biological factors in colorectal cancer is needed, particularly in relation to known CMS subtypes. The small number of genes needed to quantify metagene expression make it potentially suitable for development as a rapid diagnostic tool while its role as a canonical, cell intrinsic pathway makes it a relevant target for novel targeted therapies. 


\section{Statements}

\section{Contributions:}

Conceptualisation: SMO'C, CHW, AJL, MAH, TSM; methodology and data acquisition:

SMO'C and CHW; data curation and statistical analysis: CHW; resources: SMO'C, CHW, AJL, CCH, MAH, TSM; writing (original draft): SMO'C, CHW; writing (review and editing): SMO'C, CHW, AJL, CCH, MAH, TSM; supervision: $\mathrm{CCH}$ and TSM.

\section{Funding:}

The authors acknowledge the support of the UK Medical Research Council and Cancer Research UK stratified medicine consortium for colorectal cancer (S:CORT) in relation to collection, processing and quality control of the FOCUS trial samples, funding of CHW, and also the patients who participated in the trial. SMO'C is CRUK clinical research fellow (grant number H3R00390.H376). MAH is funded by Medical Research Council (grant number MC/PC/12001/2). Annabelle Lewis is supported by MRC (MR/P000738/1). CCH is supported by the Medical Research Council, the EPSRC, the Alan Turing Institute and the Li Ka Shing Centre for Health Innovation and Discovery. Aspects of this work have been presented in abstract form at ASCO GI 2019 symposium. 


\section{REFERENCES}

1 Bowel cancer incidence statistics. Cancer Res. UK.

2015.http://www.cancerresearchuk.org/health-professional/cancer-statistics/statistics-bycancer-type/bowel-cancer/incidence (accessed 17 Nov 2016).

2 Petrelli F, Tomasello G, Borgonovo K, et al. Prognostic Survival Associated With LeftSided vs Right-Sided Colon Cancer: A Systematic Review and Meta-analysis. JAMA Oncol 2017;3:211-9. doi:10.1001/jamaoncol.2016.4227

3 Stintzing S, Miller-Phillips L, Modest DP, et al. Impact of BRAF and RAS mutations on first-line efficacy of FOLFIRI plus cetuximab versus FOLFIRI plus bevacizumab: analysis of the FIRE-3 (AIO KRK-0306) study. Eur J Cancer 2017;79:50-60. doi:10.1016/j.ejca.2017.03.023

4 Flemer B, Lynch DB, Brown JMR, et al. Tumour-associated and non-tumour-associated microbiota in colorectal cancer. Gut 2017;66:633-43. doi:10.1136/gutjnl-2015-309595

5 Sjoquist KM, Renfro LA, Simes RJ, et al. Personalizing Survival Predictions in Advanced Colorectal Cancer: The ARCAD Nomogram Project. JNCI J Natl Cancer Inst 2018;110:638-48. doi:10.1093/jnci/djx253

6 Popat S, Hubner R, Houlston R s. Systematic Review of Microsatellite Instability and Colorectal Cancer Prognosis. J Clin Oncol 2005;23:609-18.

doi:10.1200/JCO.2005.01.086

7 Guastadisegni C, Colafranceschi M, Ottini L, et al. Microsatellite instability as a marker of prognosis and response to therapy: A meta-analysis of colorectal cancer survival data. Eur J Cancer 2010;46:2788-98. doi:10.1016/j.ejca.2010.05.009

8 Guinney J, Dienstmann R, Wang X, et al. The consensus molecular subtypes of colorectal cancer. Nat Med 2015;21:1350-6. doi:10.1038/nm.3967

9 Sanchez-Vega F, Mina M, Armenia J, et al. Oncogenic Signaling Pathways in The Cancer Genome Atlas. Cell 2018;173:321-337.e10. doi:10.1016/j.cell.2018.03.035

10 Taguchi K, Motohashi H, Yamamoto M. Molecular mechanisms of the Keap1-Nrf2 pathway in stress response and cancer evolution. Genes Cells 2011;16:123-40. doi:10.1111/j.1365-2443.2010.01473.x

11 Menegon S, Columbano A, Giordano S. The Dual Roles of NRF2 in Cancer. Trends Mol Med 2016;22:578-93. doi:10.1016/j.molmed.2016.05.002

12 Sporn MB, Liby KT. NRF2 and cancer: the good, the bad and the importance of context. Nat Rev Cancer 2012;12:564-71. doi:10.1038/nrc3278

13 Jaramillo MC, Zhang DD. The emerging role of the Nrf2-Keap1 signaling pathway in cancer. Genes Dev 2013;27:2179-91. doi:10.1101/gad.225680.113

14 Kitamura Hiroshi, Motohashi Hozumi. NRF2 addiction in cancer cells. Cancer Sci 2018;0. doi:10.1111/cas.13537 
15 Yoo NJ, Kim HR, Kim YR, et al. Somatic mutations of the KEAP1 gene in common solid cancers. Histopathology 2012;60:943-52. doi:10.1111/j.1365-2559.2012.04178.x

16 Network TCGA. Comprehensive molecular characterization of human colon and rectal cancer. Nature 2012;487:330-7. doi:10.1038/nature11252

17 Hanada N, Takahata T, Zhou Q, et al. Methylation of the KEAP1 gene promoter region in human colorectal cancer. BMC Cancer 2012;12:66. doi:10.1186/1471-2407-12-66

18 DeNicola GM, Karreth FA, Humpton TJ, et al. Oncogene-induced Nrf2 transcription promotes ROS detoxification and tumorigenesis. Nature 2011;475:106-9.

doi:10.1038/nature10189

19 Levings DC, Wang X, Kohlhase D, et al. A distinct class of antioxidant response elements is consistently activated in tumors with NRF2 mutations. Redox Biol 2018;19:235-49. doi:10.1016/j.redox.2018.07.026

20 Chibon F. Cancer gene expression signatures - The rise and fall? Eur J Cancer 2013;49:2000-9. doi:10.1016/j.ejca.2013.02.021

21 Qian Z, Zhou T, Gurguis CI, et al. Nuclear factor, erythroid 2-like 2-associated molecular signature predicts lung cancer survival. Sci Rep 2015;5. doi:10.1038/srep16889

22 Namani A, Cui QQ, Wu Y, et al. NRF2-regulated metabolic gene signature as a prognostic biomarker in non-small cell lung cancer. Oncotarget 2017;5. doi:10.18632/oncotarget.19349

23 Rhodes DR, Yu J, Shanker K, et al. ONCOMINE: A Cancer Microarray Database and Integrated Data-Mining Platform. Neoplasia N Y N 2004;6:1-6.

24 Storey JD, Tibshirani R. Statistical significance for genomewide studies. Proc Natl Acad Sci 2003;100:9440-5. doi:10.1073/pnas.1530509100

25 Davis S, Meltzer PS. GEOquery: a bridge between the Gene Expression Omnibus (GEO) and BioConductor. Bioinformatics 2007;23:1846-7. doi:10.1093/bioinformatics/btm254

26 Huber W, Carey VJ, Gentleman R, et al. Orchestrating high-throughput genomic analysis with Bioconductor. Nat Methods 2015;12:115-21. doi:10.1038/nmeth.3252

27 Smith JJ, Deane NG, Wu F, et al. Experimentally Derived Metastasis Gene Expression Profile Predicts Recurrence and Death in Patients With Colon Cancer. Gastroenterology 2010;138:958. doi:10.1053/j.gastro.2009.11.005

28 Jorissen RN, Gibbs P, Christie M, et al. Metastasis-associated gene expression changes predict poor outcomes in patients with Dukes' stage B and C colorectal cancer. Clin Cancer Res Off J Am Assoc Cancer Res 2009;15:7642-51. doi:10.1158/1078-0432.CCR09-1431

29 Marisa L, Reyniès A de, Duval A, et al. Gene Expression Classification of Colon Cancer into Molecular Subtypes: Characterization, Validation, and Prognostic Value. PLOS Med 2013;10:e1001453. doi:10.1371/journal.pmed.1001453 
30 Seymour MT, Maughan TS, Ledermann JA, et al. Different strategies of sequential and combination chemotherapy for patients with poor prognosis advanced colorectal cancer (MRC FOCUS): a randomised controlled trial. The Lancet 2007;370:143-52. doi:10.1016/S0140-6736(07)61087-3

$31 \mathrm{Hu}$ Y, Gaedcke J, Emons G, et al. Colorectal cancer susceptibility loci as predictive markers of rectal cancer prognosis after surgery. Genes Chromosomes Cancer 2018;57:140-9. doi:10.1002/gcc. 22512

32 Buuren S van, Groothuis-Oudshoorn K. mice: Multivariate Imputation by Chained Equations in R. J Stat Softw 2011;45:1-67. doi:10.18637/jss.v045.i03

33 R Core Team. R: A Language and Environment for Statistical Computing. Vienna, Austria: : R Foundation for Statistical Computing 2016. https://www.R-project.org/

34 Gaedcke J, Grade M, Jung K, et al. Mutated KRAS results in overexpression of DUSP4, a MAP-kinase phosphatase, and SMYD3, a histone methyltransferase, in rectal carcinomas. Genes Chromosomes Cancer;49:1024-34. doi:10.1002/gcc.20811

35 Graudens E, Boulanger V, Mollard C, et al. Deciphering cellular states of innate tumor drug responses. Genome Biol 2006;7:R19. doi:10.1186/gb-2006-7-3-r19

36 Hong Y, Downey T, Eu KW, et al. A 'metastasis-prone' signature for early-stage mismatch-repair proficient sporadic colorectal cancer patients and its implications for possible therapeutics. Clin Exp Metastasis 2010;27:83-90. doi:10.1007/s 10585-0109305-4

37 Kaiser S, Park Y-K, Franklin JL, et al. Transcriptional recapitulation and subversion of embryonic colon development by mouse colon tumor models and human colon cancer. Genome Biol 2007;8:R131. doi:10.1186/gb-2007-8-7-r131

38 Sabates-Bellver J, Flier LGV der, Palo M de, et al. Transcriptome Profile of Human Colorectal Adenomas. Mol Cancer Res 2007;5:1263-75. doi:10.1158/1541-7786.MCR07-0267

39 Skrzypczak M, Goryca K, Rubel T, et al. Modeling Oncogenic Signaling in Colon Tumors by Multidirectional Analyses of Microarray Data Directed for Maximization of Analytical Reliability. PLOS ONE 2010;5:e13091. doi:10.1371/journal.pone.0013091

40 Cloer EW, Goldfarb D, Schrank TP, et al. NRF2 Activation in Cancer: From DNA to Protein. Cancer Res Published Online First: 13 February 2019. doi:10.1158/00085472.CAN-18-2723

41 Rojo de la Vega M, Chapman E, Zhang DD. NRF2 and the Hallmarks of Cancer. Cancer Cell 2018;34:21-43. doi:10.1016/j.ccell.2018.03.022

42 Ji L, Wei Y, Jiang T, et al. Correlation of Nrf2, NQO1, MRP1, cmyc and p53 in colorectal cancer and their relationships to clinicopathologic features and survival. Int $J$ Clin Exp Pathol 2014;7:1124-31.

$43 \mathrm{Hu} \mathrm{T}$, Yao Y, Yu S, et al. Clinicopathologic significance of CXCR4 and Nrf2 in colorectal cancer. J Biomed Res 2013;27:283-90. doi:10.7555/JBR.27.20130069 
44 Szklarczyk D, Morris JH, Cook H, et al. The STRING database in 2017: qualitycontrolled protein-protein association networks, made broadly accessible. Nucleic Acids Res 2017;45:D362-8. doi:10.1093/nar/gkw937

45 Kang KA, Piao MJ, Kim KC, et al. Epigenetic modification of Nrf2 in 5-fluorouracilresistant colon cancer cells: involvement of TET-dependent DNA demethylation. Cell Death Dis 2014;5:e1183. doi:10.1038/cddis.2014.149

46 Malhotra D, Portales-Casamar E, Singh A, et al. Global mapping of binding sites for Nrf2 identifies novel targets in cell survival response through ChIP-Seq profiling and network analysis. Nucleic Acids Res 2010;38:5718-34. doi:10.1093/nar/gkq212 


\section{Tables / Figures}

Table 1. Cohort characteristics of the training and validation sets. The numbers of cases, type of tissue, RNA expression platform, outcome variable and available covariates for adjusted analyses are indicated. $(\mathrm{DFS}=$ Disease Free Survival, $\mathrm{OS}=$ Overall survival, $\mathrm{dMMR}=$ deficient Mismatch repair, pMMR = proficient Mismatch Repair).

\begin{tabular}{|c|c|c|c|c|c|c|}
\hline & & $\begin{array}{l}\text { GSE17536 } \\
\text { (Training } \\
\text { set) }\end{array}$ & GSE14333 & GSE39582 & $\begin{array}{l}\text { MRC FOCUS } \\
\text { trial }\end{array}$ & GSE87211 \\
\hline \multicolumn{2}{|c|}{ Patients } & 177 & 226 & 570 & $\begin{array}{l}375 \\
\text { (355 DNA } \\
\text { mutant } \\
\text { status) }\end{array}$ & 189 \\
\hline \multicolumn{2}{|c|}{ Tissue type } & Fresh frozen & Fresh frozen & Fresh frozen & $\begin{array}{l}\text { Formalin } \\
\text { fixed } \\
\text { paraffin } \\
\text { embedded }\end{array}$ & Fresh frozen \\
\hline \multicolumn{2}{|c|}{ Platform array } & $\begin{array}{l}\text { Affymetrix } \\
\text { U133 v2.0 }\end{array}$ & $\begin{array}{l}\text { Affymetrix } \\
\text { U133 v2.0 }\end{array}$ & $\begin{array}{l}\text { Affymetrix } \\
\text { U133 v2.0 }\end{array}$ & $\begin{array}{l}\text { Affymetrix } \\
\text { Xcel }\end{array}$ & $\begin{array}{l}\text { Agilent } \\
\text { Human } 4 \mathrm{x} \\
44 \mathrm{k} \text { v2 }\end{array}$ \\
\hline \multicolumn{2}{|c|}{ Primary site } & Colon & Colorectal & Colon & Colon & Rectum \\
\hline \multirow[t]{4}{*}{ Stage } & I & $24(13.6 \%)$ & $41(18 \%)$ & $37(6.5 \%)$ & & \\
\hline & II & $57(32.2 \%)$ & $95(42 \%)$ & $267(47 \%)$ & & $70(30.8 \%)$ \\
\hline & III & $57(32.2 \%)$ & $93(41 \%)$ & $206(36 \%)$ & & $143(63 \%)$ \\
\hline & IV & $39(22 \%)$ & & $60(10.5 \%)$ & $375(100 \%)$ & $14(6.2 \%)$ \\
\hline \multicolumn{2}{|c|}{ Outcome variable } & OS & DFS & $\begin{array}{l}\text { DFS } \\
\text { OS }\end{array}$ & OS & $\begin{array}{l}\text { DFS } \\
\text { OS }\end{array}$ \\
\hline \multicolumn{7}{|c|}{ Covariates } \\
\hline \multirow{2}{*}{\multicolumn{2}{|c|}{ Chemo(radio)therapy }} & & Yes 72 & Yes 240 & $375(100 \%)$ & $189(100 \%)$ \\
\hline & & & No 154 & No 326 & & \\
\hline \multirow{2}{*}{\multicolumn{2}{|c|}{ Site of primary }} & & & Prox. 232 & Left 203 & \\
\hline & & & & Dist. 351 & Right 152 & \\
\hline \multicolumn{2}{|c|}{$\begin{array}{l}\text { BRAF V600E } \\
\text { mutation }\end{array}$} & & & Mut 51 & $38(10 \%)$ & \\
\hline \multirow{2}{*}{\multicolumn{2}{|c|}{$\begin{array}{l}\text { Mismatch Repair } \\
\text { status }\end{array}$}} & & & dMMR 77 & dMMR 15 & \\
\hline & & & & pMMR 459 & pMMR 326 & \\
\hline
\end{tabular}


Figure 1. A) Aggregate heatmaps for Oncomine database over all over expressed and under expressed genes in 9 different colorectal datasets. The median rank for the meta-analysis and the corrected p-value are shown. 40 of the 62 candidate genes are significantly overexpressed and/or underexpressed in colorectal cancer relative to normal tissue at a FDR $<0.05 .36$ of these were represented in the GSE17536, after filtering by probe-matching, so were taken forward for further exploration. B) Pairwise correlation heatmap showing the degree of positive (red) and negative (blue) Pearson correlation between the 36 genes of the NRF2 pathway in the training set. The high positive correlation between a subgroup of genes (top right) indicates a high degree of co-expression.

A

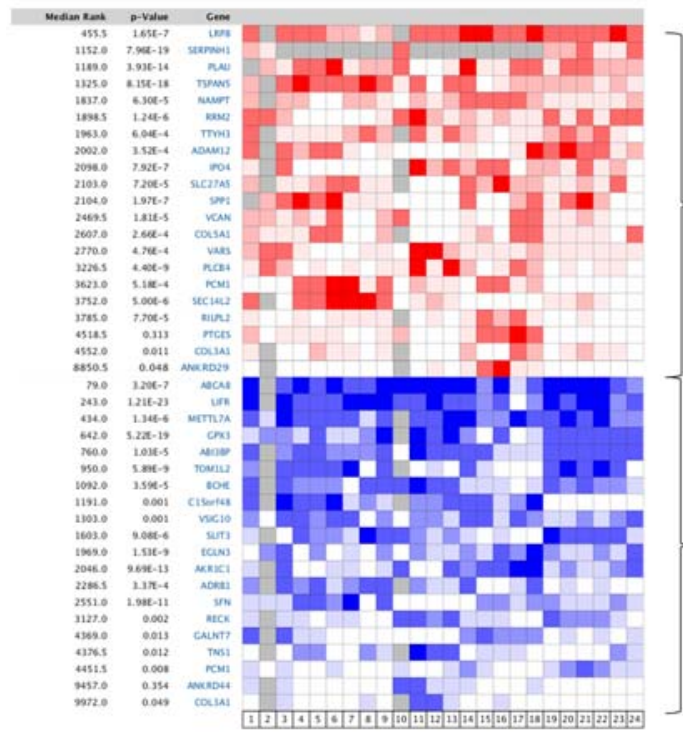

B

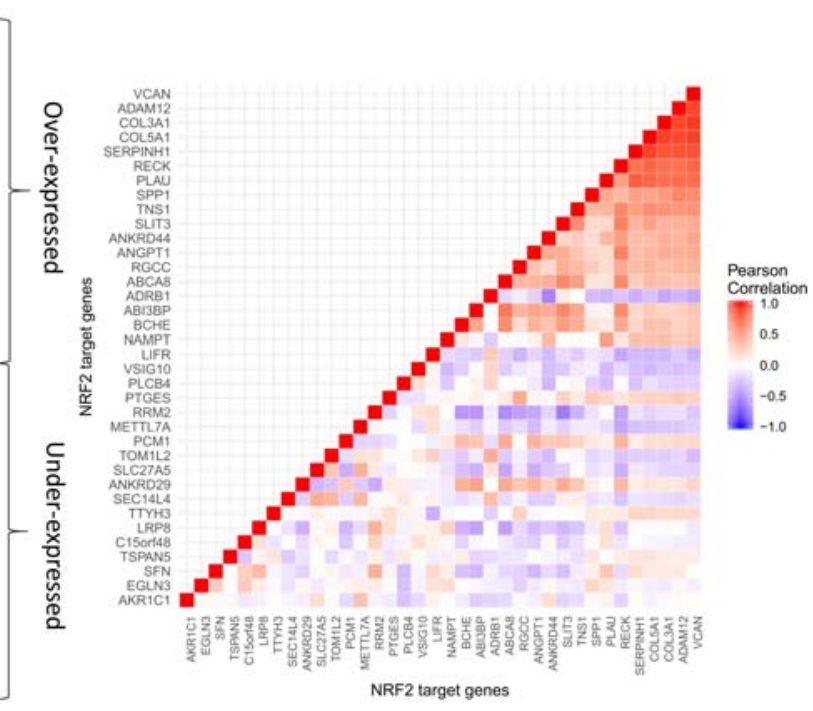


Figure 2. Kaplan-Meier curves and associated risk tables for the primary analysis of three datasets. All show survival outcomes for patients with high, intermediate and low (induced by tertiles) of NRF2 metagene expression. A) GSE14333 and B) GSE39582 represents early stage I-III patients C ) GSE39582 represents stage I-IV patients and C) MRC FOCUS represents stage IV first line metastatic patients. For the Kaplan Meier curves, a median cut point was used to binarise NRF2 metagene expression.

A GSE14333

Strata + NRF2 low + NRF2 median + NRF2 high

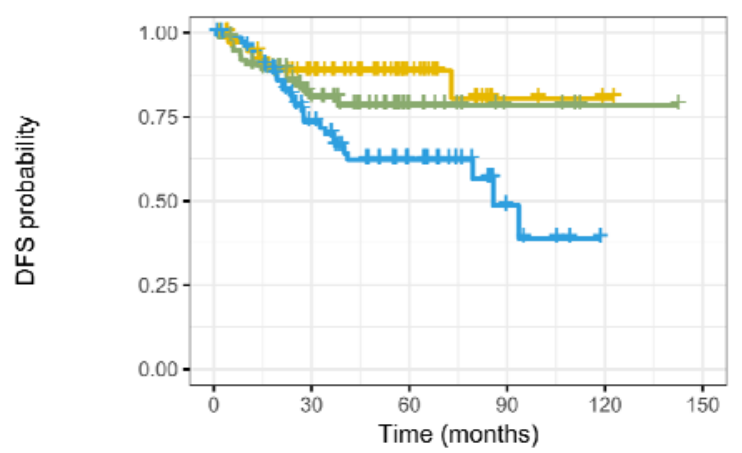

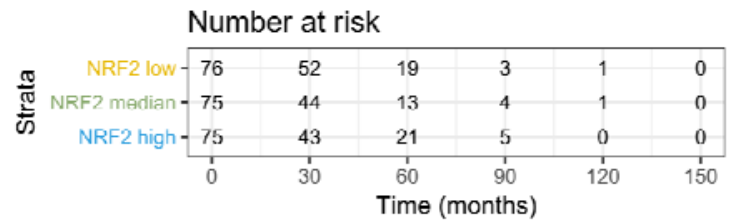

C

$$
\text { GSE39582 (OS) }
$$

Strata + NRF low + NRF2 medium + NRF2 high

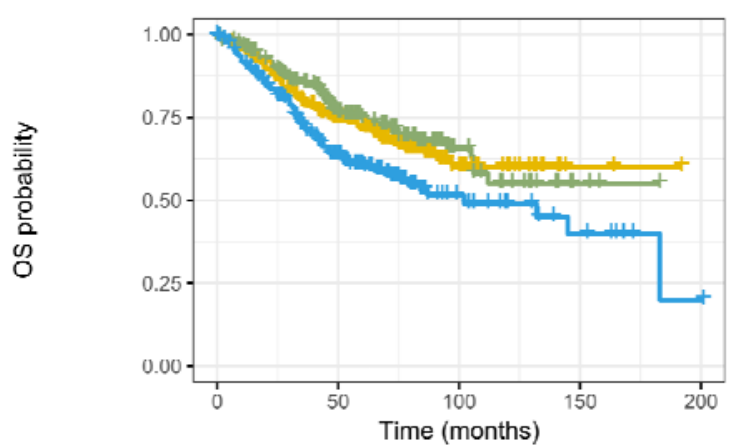

Number at risk

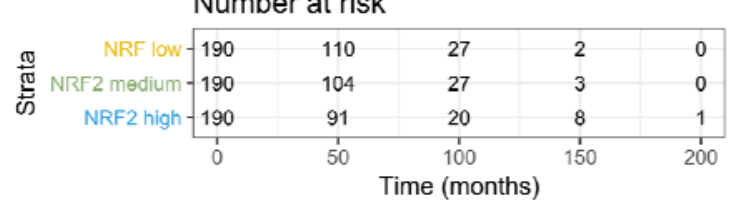

B

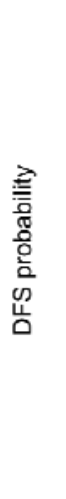

D
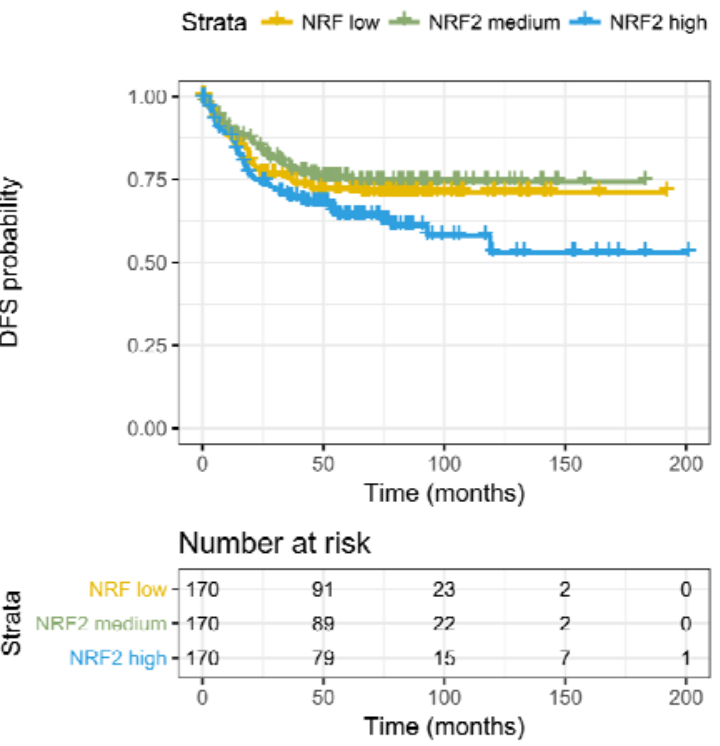

\section{MRC FOCUS}

Strata + NRF2 low + NRF2 median + NRF2 high
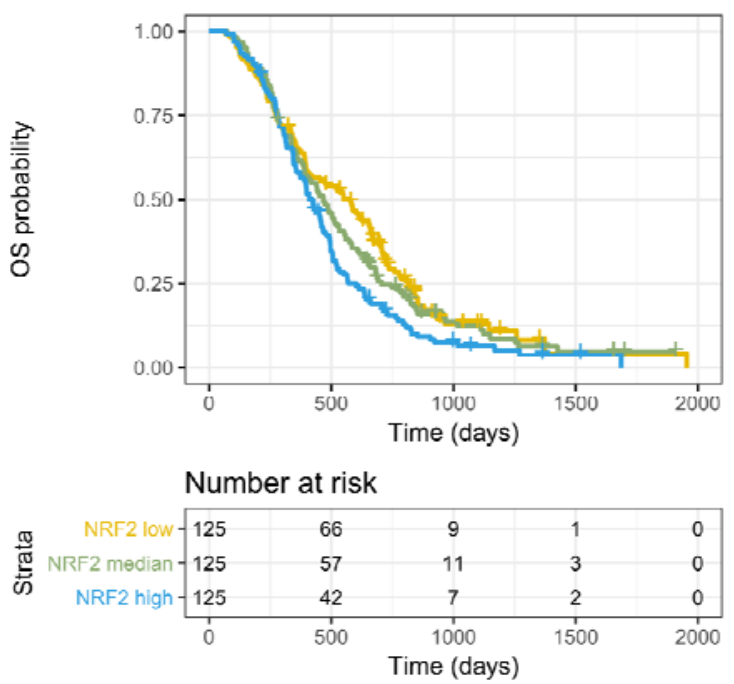
Figure 3. Kaplan Meier curves and associated risk tables for the primary analyses of

GSE87211. On the left, high NRF2 metagene expression is associated with worse disease free survival with persistent separation of the curves. On the right, there was no effect on overall survival. For the Kaplan Meier curves, high, intermediate and low (tertiles) of NRF2 expression was used.

A

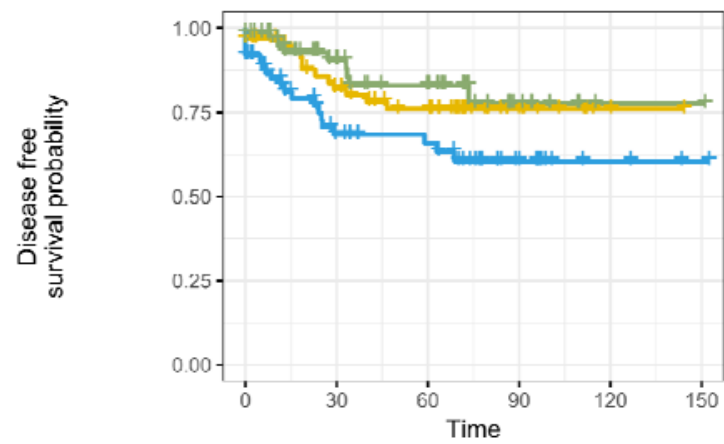

B

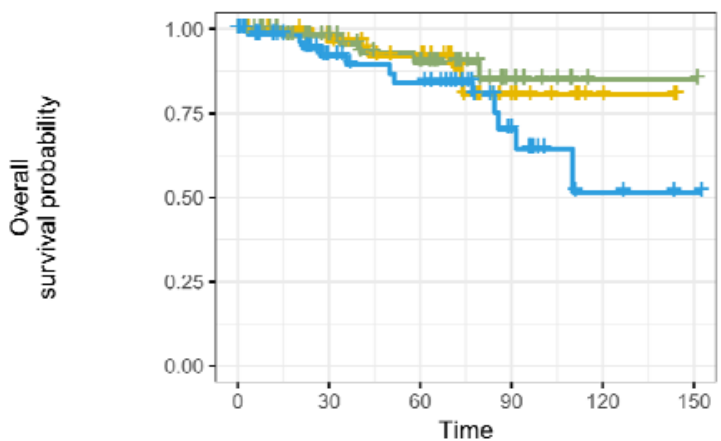

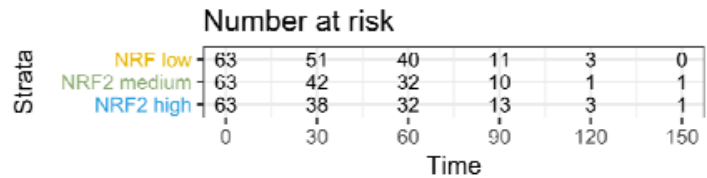


Table 2. Summary table of the Cox proportional hazard model analyses showing the numbers of patients included in each analysis, the adjusting variables where used and the p-value for the Cox model comparison $(\mathrm{LRT}=$ Likelihood ratio test $)$

\begin{tabular}{|c|c|c|c|c|c|}
\hline & $\mathbf{N}$ & Analysis & Variables & $\begin{array}{l}\mathrm{HR}^{*} \\
\text { (C.I) }\end{array}$ & $\begin{array}{c}\text { LRT } \\
\text { (p-value) }\end{array}$ \\
\hline \multicolumn{6}{|l|}{ Data set } \\
\hline \multirow[t]{2}{*}{ GSE14333 } & 226 & Primary & N/A & $\begin{array}{c}1.113 \\
(1.045-1.184)\end{array}$ & 0.0008 \\
\hline & & Secondary & $\begin{array}{l}\text { Stage } \\
\text { Adjuvant } \\
\text { chemotherapy }\end{array}$ & $\begin{array}{c}1.079 \\
(1.012-1.150)\end{array}$ & 0.0201 \\
\hline \multirow[t]{2}{*}{ MRC FOCUS } & 375 & Primary & N/A & $\begin{array}{c}1.033 \\
(1.009-1.245)\end{array}$ & 0.008 \\
\hline & & Secondary & $\begin{array}{l}\text { Sidedness } \\
\text { BRAF V600E } \\
\text { mutation }^{¥}\end{array}$ & $\begin{array}{c}1.029 \\
(1.005-1.054)\end{array}$ & 0.0185 \\
\hline \multirow[t]{2}{*}{$\begin{array}{l}\text { GSE39582 } \\
\text { (OS) }\end{array}$} & 570 & Primary & N/A & $\begin{array}{c}1.054 \\
(1.02-1.089)\end{array}$ & 0.001 \\
\hline & & Secondary & Stage & $\begin{array}{c}1.042 \\
(1.010-1.076)\end{array}$ & 0.01 \\
\hline \multirow[t]{2}{*}{$\begin{array}{l}\text { GSE39582 } \\
\text { (DFS) }\end{array}$} & 510 & Primary & N/A & $\begin{array}{c}1.04 \\
(1.002-1.08)\end{array}$ & 0.0383 \\
\hline & & Secondary & $\begin{array}{l}\text { Stage } \\
\text { Mismatch repair } \\
(\mathrm{MMR})^{¥}\end{array}$ & $\begin{array}{c}1.039 \\
(1.000-1.080)\end{array}$ & 0.049 \\
\hline $\begin{array}{l}\text { GSE87211 } \\
\text { (DFS) }\end{array}$ & 189 & Primary & N/A & $\begin{array}{c}1.127 \\
(1.019-1.245)\end{array}$ & 0.056 \\
\hline $\begin{array}{l}\text { GSE87211 } \\
\text { (OS) }\end{array}$ & 189 & Primary & N/A & $\begin{array}{c}1.135 \\
(0.985-1.309)\end{array}$ & 0.197 \\
\hline
\end{tabular}

* hazard ratio for event per unit increase in expression of NRF2 metagene (continuous variable) which is different to HR between the upper and lower tertiles

$¥$ missing variables imputed 
Figure 4. CMS classification was derived for the MRC FOCUS trial dataset. The barplot shows the proportion of high and low NRF2 metagene expression in each of the four CMS subtypes. CMS 4 was substantially enriched for high Nrf2 expression.

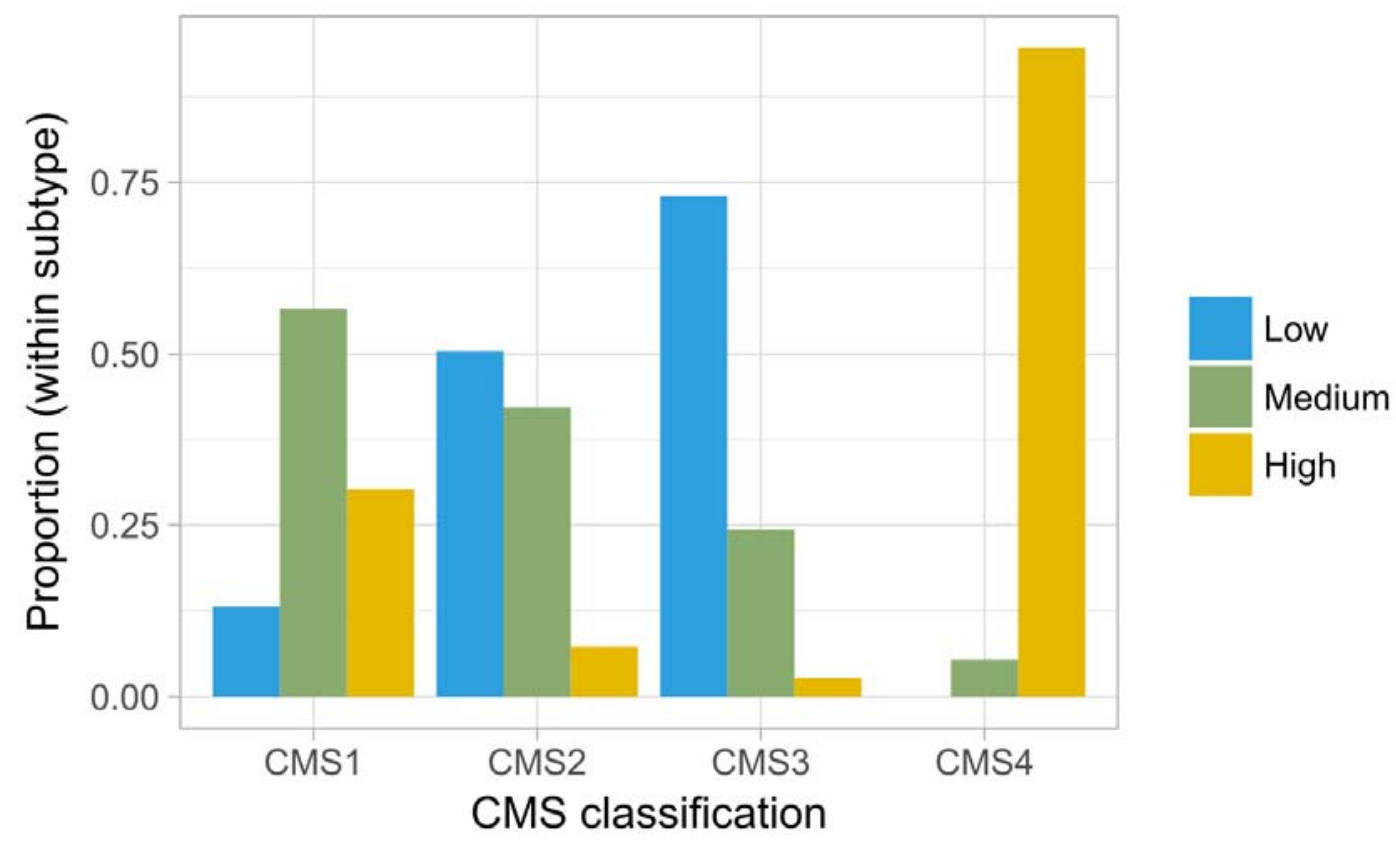




\section{Supplementary Information}

\section{Unsupervised dimension reduction}

The metagene approach has the advantage of preserving statistical power. If all the candidate genes were included in an analysis, the statistical power would be exhausted. In addition, it was expected that some probes would be correlated due to multiple probes matching to the same gene symbol and co-expression of genes in the pathway. Such correlations create multicollinearity in the downstream regression analysis, which leads to instability in parameter estimation. It was therefore desirable to obtain a simplified aggregate of NRF2 representation, which was achieved by applying principal component analysis (PCA) to the candidate NRF2 target genes.

PCA produced a new set of continuous variables, which are weighted averages of all the probe expressions. In the presence of high correlation among probes, the majority of the variation in the data can be explained by a smaller set of principal components (PCs). Thus PCA can provide a simpler representation of the expression data.

Specifically, we filtered out PCs that explained too little variation in the expression data and only retained the "major PCs", which were the most variable PCs that explained up to $80 \%$ of the variation in the expression data. Such filter was applied because each of the excluded PCs would represent too little variation and would roughly be the same amount as that explained by other excluded PCs. Often, such minor PCs would have arbitrary probe weights, so should not be interpreted.

Furthermore, the PCs are uncorrelated among themselves, which eliminated the problem of multi-collinearity in the regression analysis downstream. 


\section{Supervised selection of the principal components}

Inclusion of major PCs that have little or no explanatory power can dilute the signal in an analysis. Therefore, supervised variable selection was performed to indicate how many major PCs were useful for and hence should be kept for prognosis prediction.

The Cox proportional hazard regression was used to model the prognosis prediction by the PCs of NRF2 expression. For variable selection, we progressively included PCs in decreasing order of variance. Akaike information criteria (AIC) and Bayesian information criteria (BIC) were calculated to indicate the most suitable number of major PCs to keep. The selected set of major PCs were used to represent the NRF2 metagene derived from the training set.

Prior to validation, we identified the genes that were represented by the most predictive PCs to understand the biological signal in the training set. If the absolute correlation of a PC and a gene's probe expression was $>0.5$, then that $\mathrm{PC}$ would be considered to be representative of the gene of interest. For convenience, these genes are collectively referred to as the 'represented gene set'. While a PC is a weighted average of the probe expressions of all the target genes that does not mean all the probes would be correlated with the PC. Therefore, the represented gene set would likely be a subset of the target genes.

In the training set, PC1 was the consensus between $\mathrm{AIC}$ and $\mathrm{BIC}$. The represented gene set of PC1 were VCAN, ADAM12, COL3A1, COL5A1, SERPINH1, RECK, PLAU, SPPI, TNS1 and SLIT3. These 10 genes were therefore deemed the best biological representation of the pathway that explained the survival outcome. Hence that was the pattern that should be sought following PCA in the validation sets, in order to ensure that we were validating the biological signal that was consistent with that discovered in the training set. 


\section{Construction of the NRF2 metagene in the validation sets}

The NRF2 metagene in each validation set was obtained by performing PCA on the corresponding probe sets. Major PCs were identified using the same filtering method for the training set. It is worth noting that the PCs are unsupervised summary statistics of the probe expressions. Thus, which PCs correlated with the probe expressions of a given gene could vary purely due to sampling variation. Specifically, while PC1 of the training set most strongly correlated with the represented gene set, a different set of PCs of a validation set might most strongly correlate with the represented gene set (derived from the training set). In that case, it would not be appropriate to use a fix set of PCs to validate the biological signal discovered in the training set.

For a given validation set, the correlations between the major PCs and the probe expressions of genes in the represented set were calculated. Based on the PC-probe correlations, PCs were selected to represent the metagene if those PCs were representative of any genes in the represented gene set. As discussed, the number of PCs selected via this approach could vary across datasets.

\section{RNA expression of MRC FOCUS}

S:CORT undertook multiplexed analysis of a subset of FFPE primary tumour samples from the FOCUS trial (NCT00008060), which included RNA microarray analysis on the Affymetrix Xcel array. Samples were hybridised to the Affymetrix Xcel microarray as per the manufacturer's instructions. Quality control analysis was run on samples using the R base 'AffyQC' module (https://github.com/BiGCAT-UM/affyQCModule). The files were then processed using the R packages 'limma' https://bioconductor.org.packages/release/bioc/html/limma.html) and 'affy' (https://bioconductor.org.packages/release/bioc/html/affy.html) to normalise the expression 
values using robust multiarray algorithm (RMA) and generate and expression matrix of probe intensities against the samples. CMS subtypes were determined from gene expression microarray data using the CMS classifier package in R (https://github.com/SageBionetworks/CMSclassifier).

\section{Expression arrays and probe matching}

To minimise differences between datasets induced by different platforms of RNA profiling, gene symbols were matched to probesets from the expression array annotation file, often resulting in multiple probes per gene. Probes were matched between platforms using genomic locus references from the annotation file (see supplementary table 2 - 4).

The RNA expression of GSE1433 and GSE39582 validation set was measured by the same platform, Affymetrix U133 v2, as that used in the training set so all probes could be used. However, the MRC FOCUS and GSE87211validation sets were respectively measured using the Affymetrix Xcel and Agilent Human 4 x 44K v2. In order to ensure that the metagene derived from the training can be translated to the IV validation set, we identified the U133 probes that matched to the Xcel and Agilent probes. Two types of matches were considered-(i) exact-match and (ii) sub/super-set-match. Exact match was where the chromosomal alignment from the annotation file of the microarray build probes matched perfectly. Sub/super-set-match occurred where the U133 probe was completely nested in an Xcel probe or vice versa. The same scheme was applied to matching to Agilent probes. In total the 36 genes were represented by 92 probes in Affymetrix U133 v2, 140 probes in Affymetrix Xcel array and 46 probes in Agilent Human 4 x 44K v2 array. Unmatched probes were filtered out prior to analysis. The 92 probe set in GSE17536 was used as the training dataset of this study. 


\section{Regulatory sequence analysis}

To further understand the role of NRF2 in regulating the represented gene set from the selected PCA in the training, in silico analysis of putative AREs in metabolic gene promoters were identified using RSAT [1]. The promoter sequence upstream to $-5 \mathrm{~kb}$ lengths for named genes were retrieved from Eukaryotic Promoter Database (EPD) [2]. Subsequently, these sequences were analysed to identify AREs using the string-based pattern matching RSAT program 'dna pattern' [3]. The DNA patterns were entered as RTGASNNNGCR and RTGAYNNNGCR, where $\mathrm{R}=\mathrm{A}$ or $\mathrm{G}, \mathrm{S}=\mathrm{C}$ or $\mathrm{G}, \mathrm{Y}=\mathrm{C}$ or $\mathrm{T}$, and $\mathrm{N}=$ any nucleotide. These generic sequence queries were used in the query option to identify an exact match of an ARE sequence in the given 5-kb promoter sequences. The DNA patterns have been used previously [3] and are based on well-known previous publications on the consensus structure of AREs [4,5]. The analysis was carried out for VCAN, ADAM12, COL3A1, COL5A1, SERPINH1, RECK, PLAU, SPPI, TNS1 and SLIT3.

\section{References}

1 Medina-Rivera A, Defrance M, Sand O, et al. RSAT 2015: Regulatory Sequence Analysis Tools. Nucleic Acids Res 2015;43:W50-6. doi:10.1093/nar/gkv362

2 Dreos R, Ambrosini G, Périer RC, et al. The Eukaryotic Promoter Database: expansion of EPDnew and new promoter analysis tools. Nucleic Acids Res 2015;43:D92-6. doi:10.1093/nar/gku1111

3 Abdullah A, Kitteringham NR, Jenkins RE, et al. Analysis of the role of Nrf2 in the expression of liver proteins in mice using two-dimensional gel-based proteomics. Pharmacol Rep 2012;64:680-97.

4 Motohashi H, O'Connor T, Katsuoka F, et al. Integration and diversity of the regulatory network composed of Maf and CNC families of transcription factors. Gene 2002;294:112. doi:10.1016/S0378-1119(02)00788-6

5 Rushmore TH, Morton MR, Pickett CB. The antioxidant responsive element. Activation by oxidative stress and identification of the DNA consensus sequence required for functional activity. J Biol Chem 1991;266:11632-9. 
Supplementary Table 1. Description and source of the datasets selected in the Oncomine database to assess differential expression of the candidate genes

\begin{tabular}{|c|c|}
\hline 1. Rectal Adenocarcinoma vs. Normal & Gaedcke Colorectal, Genes Chromosomes Cancer, 2010 \\
\hline 2. Colorectal Carcinoma vs. Normal & Graudens Colon, Genome Biol, 2006 \\
\hline 3. Colorectal Carcinoma vs. Normal & Hong Colorectal, Clin Exp Metastasis, 2010 \\
\hline 4. Cecum Adenocarcinoma vs. Normal & Kaiser Colon, Genome Biol, 2007 \\
\hline 5. Colon Adenocarcinoma vs. Normal & Kaiser Colon, Genome Biol, 2007 \\
\hline 6. Colon Mucinous Adenocarcinoma vs. Normal & Kaiser Colon, Genome Biol, 2007 \\
\hline 7. Rectal Adenocarcinoma vs. Normal & Kaiser Colon, Genome Biol, 2007 \\
\hline 8. Rectal Mucinous Adenocarcinoma vs. Normal & Kaiser Colon, Genome Biol, 2007 \\
\hline 9. Rectosigmoid Adenocarcinoma vs. Normal & Kaiser Colon, Genome Biol, 2007 \\
\hline 10. Colon Adenocarcinoma vs. Normal & Ki Colon, Int J Cancer, 2007 \\
\hline 11. Colon Adenoma vs. Normal & Sabates-Bellver Colon, Mol Cancer Res, 2007 \\
\hline 12. Rectal Adenoma vs. Normal & Sabates-Bellver Colon, Mol Cancer Res, 2007 \\
\hline 13. Colorectal Adenocarcinoma vs. Normal & Skrzypczak Colorectal, PLoS One, 2010 \\
\hline 14. Colorectal Carcinoma vs. Normal & Skrzypczak Colorectal, PLoS One, 2010 \\
\hline 15. Colon Adenoma Epithelia vs. Normal & Skrzypczak Colorectal 2, PLoS One, 2010 \\
\hline 16. Colon Adenoma vs. Normal & Skrzypczak Colorectal 2, PLoS One, 2010 \\
\hline 17. Colon Carcinoma Epithelia vs. Normal & Skrzypczak Colorectal 2, PLoS One, 2010 \\
\hline 18. Colon Carcinoma vs. Normal & Skrzypczak Colorectal 2, PLoS One, 2010 \\
\hline 19. Cecum Adenocarcinoma vs. Normal & TCGA Colorectal, Nature, 2012 \\
\hline 20. Colon Adenocarcinoma vs. Normal & TCGA Colorectal, Nature, 2012 \\
\hline 21. Colon Mucinous Adenocarcinoma vs. Normal & TCGA Colorectal, Nature, 2012 \\
\hline 22. Rectal Adenocarcinoma vs. Normal & TCGA Colorectal, Nature, 2012 \\
\hline 23. Rectal Mucinous Adenocarcinoma vs. Normal & TCGA Colorectal, Nature, 2012 \\
\hline 24. Rectosigmoid Adenocarinoma vs. Normal & TCGA Colorectal, Nature, 2012 \\
\hline
\end{tabular}


Supplementary Figure 1. Flowchart describing A) training: the process of deriving the

NRF2 metagene for prognosis prediction and B) validation: the process of constructing the

NRF2 metagene for validation of its prognostic effect.

A

\section{Training}

T1. Perform PCA to the probes mapped to the NRF2 target genes in the training set (GSE17536).

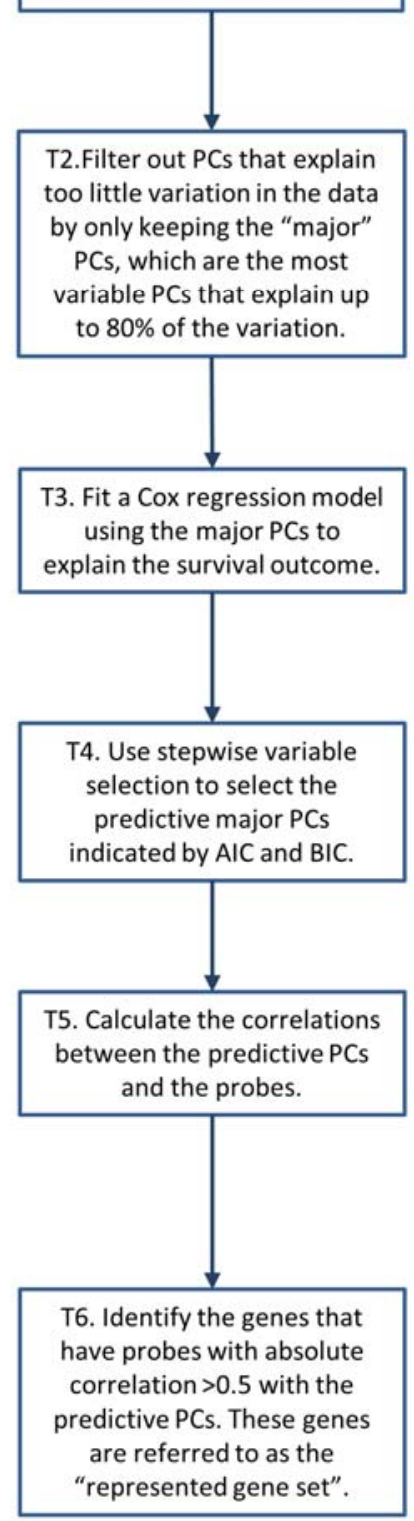

B

\section{Validation}

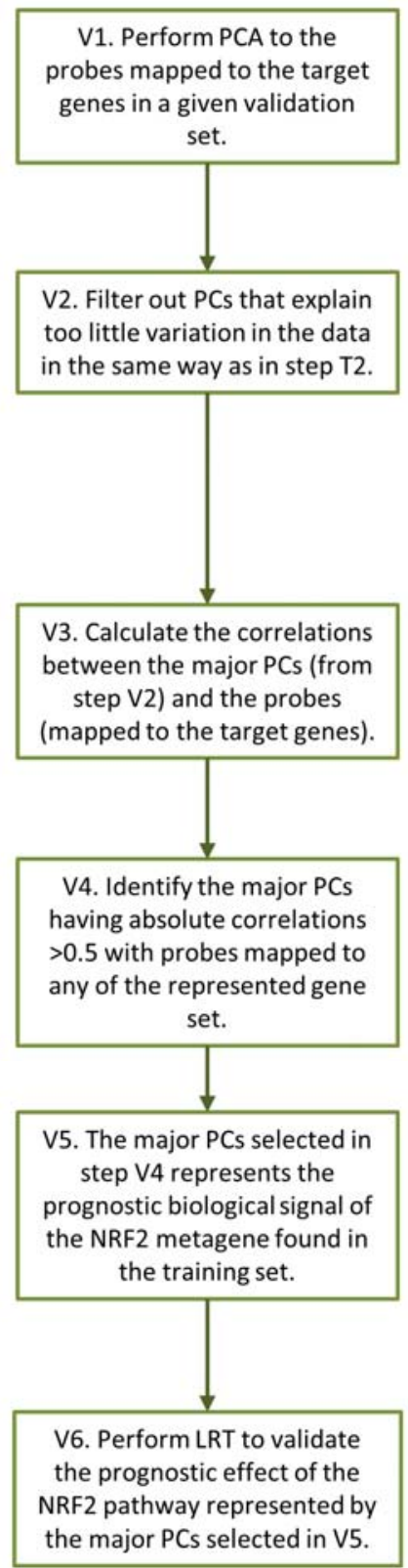


Supplementary Figure 2. Forest plot showing the relative effects of the available prognostic variables within each dataset used in the validation analyses. These variables were used in the multivariate analysis with the NRF2 metagene.

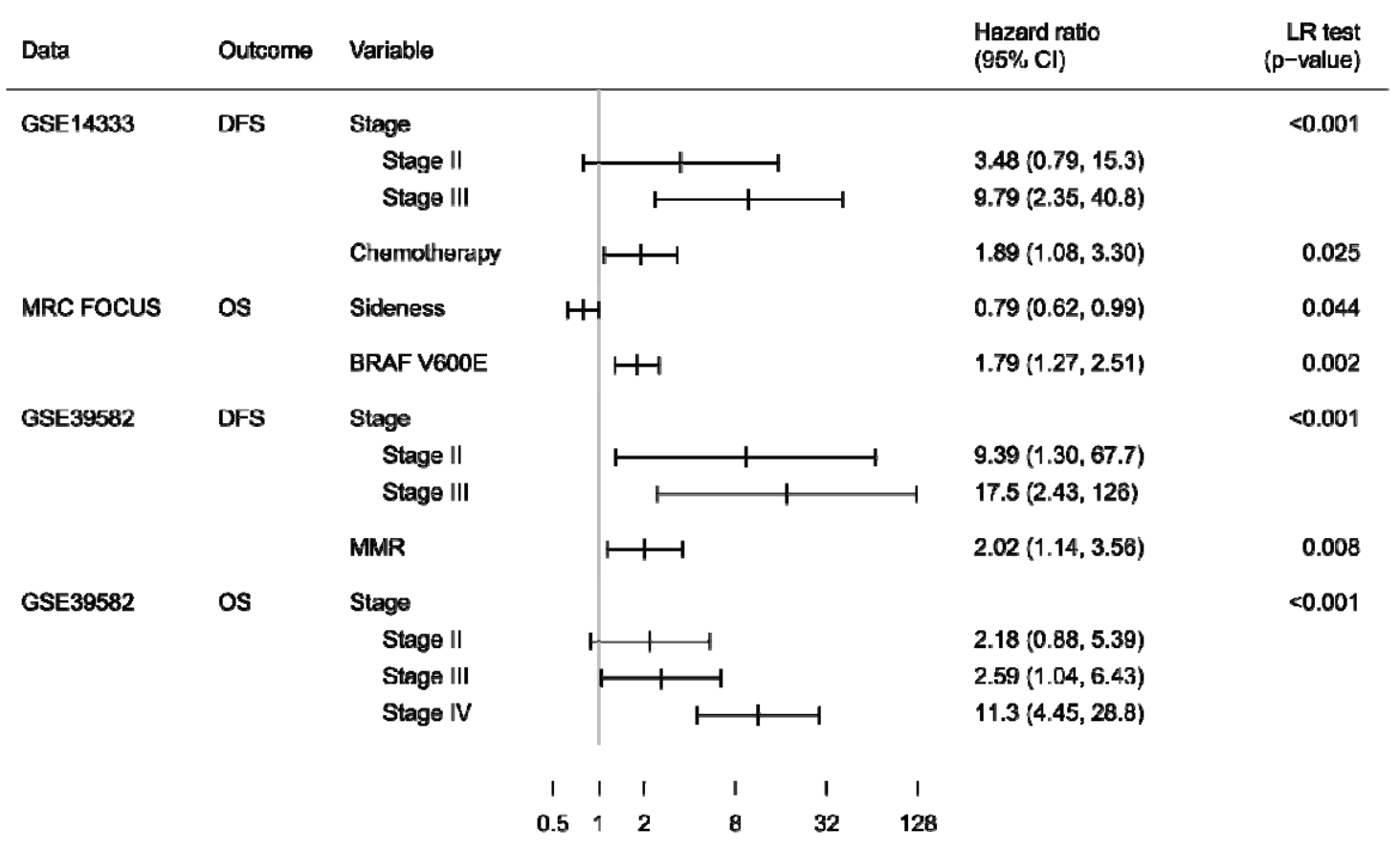


Supplementary Figure 3. Known and predicted protein-protein interactions from the STRING database for the initial 40 genes from the refined differential expression analysis.

The central network of genes (VCAN, COL3A1, COL5A1, SERPINH1, RECK, PLAU, SPPI, TNS1 etc) which show a high degree of connectivity are highly co-expressed in the training set. The line colour indicates the type of interaction and the thickness indicates the strength of interaction $($ thicker $=$ stronger $) .[$ Cyan $=$ known interaction from curated databases; purple $=$ experimentally determined; green = gene neighbourhood; red = gene fusions; dark blue $=$ gene co-occurrences; light green $=$ textmining; black $=$ co-expression; light blue $=$ protein homology]

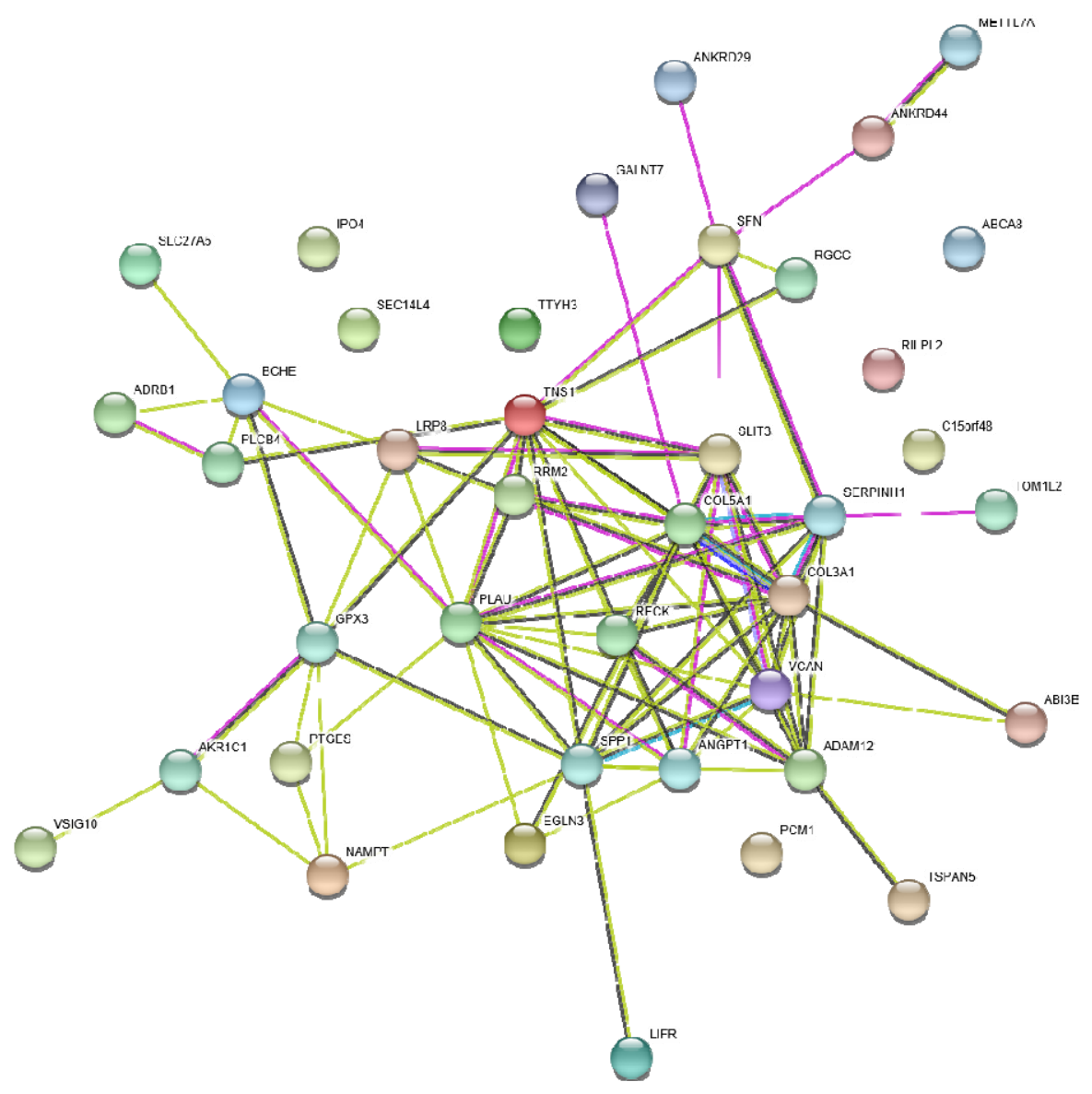


Supplementary Figure 4. Regulatory sequence analysis of the 5000 bp region upstream of the start site of the representative 10 genes which have a high correlation with PC1 in the training set. 9 of these genes contain consensus ARE sites for NRF2 to bind and directly regulate gene transcription.

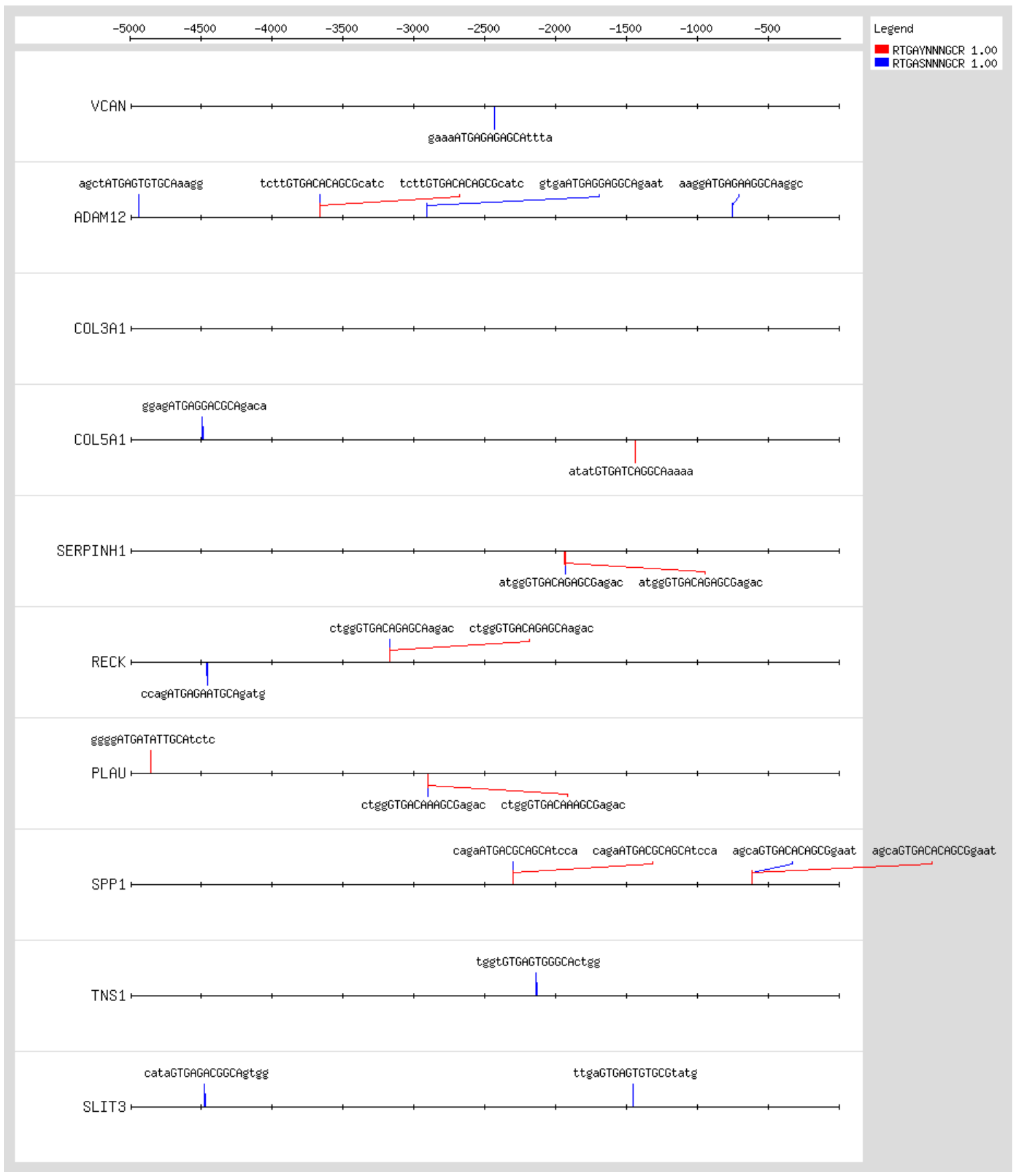


Supplementary Table 2. The list of Affymetrix U133 v2.0 probes used in the analysis and the genes to which they map

\begin{tabular}{|c|c|c|c|c|c|}
\hline Probe ID & Gene & Probe ID & Gene & Probe ID & Gene \\
\hline 1565778_at & ABCA8 & 211424_x_at & METTL7A & 1568574_x_at & SPP1 \\
\hline 204719_at & ABCA8 & 244376_at & METTL7A & 209875_s_at & SPP1 \\
\hline 220518_at & ABI3BP & 207761_s_at & METTL7A & 218863_s_at & TNS1 \\
\hline 223395_at & ABI3BP & 209703_x_at & METTL7A & 221246_x_at & TNS1 \\
\hline 1559077_at & ABI3BP & 217739_s_at & NAMPT & 214840_at & TOM1L2 \\
\hline 202952_s_at & ADAM12 & 1555167_s_at & NAMPT & 226198_at & TOM1L2 \\
\hline 204943_at & ADAM12 & 217738_at & NAMPT & 237109_at & TOM1L2 \\
\hline 215613_at & ADAM12 & 243296_at & NAMPT & 209890_at & TSPAN5 \\
\hline 213790_at & ADAM12 & 202174_s_at & PCM1 & 225387_at & TSPAN5 \\
\hline 226777_at & ADAM12 & 209996_x_at & PCM1 & 225388_at & TSPAN5 \\
\hline 208214_at & ADRB1 & 209997_x_at & PCM1 & 224674_at & TTYH3 \\
\hline 229277_at & ADRB1 & 214937_x_at & PCM1 & 204620_s_at & VCAN \\
\hline 229309_at & ADRB1 & 214118_x_at & PCM1 & 215646_s_at & VCAN \\
\hline 217626_at & AKR1C1 & 228905_at & PCM1 & 204619_s_at & VCAN \\
\hline 205608_s_at & ANGPT1 & 211668_s_at & PLAU & 211571_s_at & VCAN \\
\hline 1552939_at & ANGPT1 & 205479_s_at & PLAU & 221731_x_at & VCAN \\
\hline 205609_at & ANGPT1 & 203896_s_at & PLCB4 & 223845_at & VSIG10 \\
\hline 229308_at & ANKRD29 & 203895_at & PLCB4 & & \\
\hline 238332_at & ANKRD29 & 207388_s_at & PTGES & & \\
\hline 228471_at & ANKRD44 & 210367_s_at & PTGES & & \\
\hline 233361_at & ANKRD44 & 1558115_at & RECK & & \\
\hline 226641_at & ANKRD44 & 1558116_x_at & RECK & & \\
\hline 235777_at & ANKRD44 & 216156_at & RECK & & \\
\hline 235778_s_at & ANKRD44 & 205407_at & RECK & & \\
\hline 205433_at & BCHE & 218723_s_at & RGCC & & \\
\hline 223484_at & C15orf48 & 239827_at & RGCC & & \\
\hline 232458_at & COL3A1 & 201890_at & RRM2 & & \\
\hline 201852_x_at & COL3A1 & 209773_s_at & RRM2 & & \\
\hline 215076_s_at & COL3A1 & 216402_at & SEC14L4 & & \\
\hline 203325_s_at & COL5A1 & 207714_s_at & SERPINH1 & & \\
\hline 219232_s_at & EGLN3 & 209260_at & SFN & & \\
\hline 222847_s_at & EGLN3 & 33322_i_at & SFN & & \\
\hline 227771_at & LIFR & 33323_r_at & SFN & & \\
\hline 205876_at & LIFR & 234948_at & SLC27A5 & & \\
\hline 229185_at & LIFR & 203813_s_at & SLIT3 & & \\
\hline 208433_s_at & LRP8 & 216216_at & SLIT3 & & \\
\hline 205282_at & LRP8 & 203812_at & SLIT3 & & \\
\hline 228955_at & LRP8 & & & & \\
\hline
\end{tabular}


Supplementary Table 3. The list of Affymetrix Xcel array probes used in the analysis and the genes to which they map

\begin{tabular}{|c|c|c|c|}
\hline Probe ID & Gene & Probe ID & Gene \\
\hline ADXECAD.4694_at & ABCA8 & ADXEC.24209.C1_at & ANKRD44 \\
\hline ADXECAD.4694_x_at & ABCA8 & ADXECADA.8703_at & ANKRD44 \\
\hline ADXECEMUTR.2314_at & ABCA8 & ADXECAD.6885_at & ANKRD44 \\
\hline ADXEC.34095.C1_at & ABCA8 & ADXEC.32445.C1_s_at & ANKRD44 \\
\hline ADXEC.34095.C1_x_at & ABCA8 & ADXECEMUTR.1806_at & ANKRD44 \\
\hline ADXECAD.2828_at & ABI3BP & ADXECRS.10687_x_at & ANKRD44 \\
\hline ADXECADA.22250_at & $\mathrm{ABI} 3 \mathrm{BP}$ & ADXEC.20524.C1_at & $\mathrm{BCHE}$ \\
\hline ADXECADA.24689_s_at & ABI3BP & ADXEC.5762.C1_at & C15orf48 \\
\hline ADXECADA.13221_at & ADAM12 & ADXEC.10342.C1_at & COL3A1 \\
\hline ADXECADA.16091_at & ADAM12 & ADXEC.1138.C6_s_at & COL3A1 \\
\hline ADXECADA.19748_at & ADAM12 & ADXECAD.16292_at & COL3A1 \\
\hline ADXECADA.22926_at & ADAM12 & ADXECNTDJ.8556_at & COL3A1 \\
\hline ADXEC.10435.C1_at & ADAM12 & ADXEC.1138.C8-a_s_at & COL3A1 \\
\hline ADXEC.24608.C1_at & ADAM12 & ADXEC.1138.C8_s_at & COL3A1 \\
\hline ADXECAD.23192_s_at & ADRB1 & ADXEC.2639.C2_s_at & COL5A1 \\
\hline ADXECRS.43563_s_at & ADRB 1 & ADXECEMUTR.7334_at & COL5A1 \\
\hline ADXEC.10477.C1_s_at & ADRB1 & ADXEC.14436.C1_s_at & EGLN3 \\
\hline ADXECADA.7121_s_at & ADRB1 & ADXEC.30477.C1-a_s_at & EGLN3 \\
\hline ADXECEMUTR.4343_at & ADRB1 & ADXEC.30477.C1_at & EGLN3 \\
\hline ADXECEMUTR.4343_s_at & ADRB1 & ADXEC.9508.C1_at & EGLN3 \\
\hline ADXEC.1215.C1_x_at & AKR1C1 & ADXECNTDJ.1518_s_at & LIFR \\
\hline ADXEC.34313.C1_s_at & ANGPT1 & ADXECNTDJ.8595_at & LIFR \\
\hline ADXECAD.17898_at & ANGPT1 & ADXEC.28103.C1_s_at & LIFR \\
\hline ADXECEMUTR.2049_at & ANGPT1 & ADXECAD.15818_at & LIFR \\
\hline ADXECEMUTR.2049_x_at & ANGPT1 & ADXEC.17620.C1_at & LRP8 \\
\hline ADXECAD.22091_at & ANGPT1 & ADXEC.17620.C1_x_at & LRP8 \\
\hline ADXECNTDJ.6462_s_at & ANGPT1 & ADXECAD.26545_at & LRP8 \\
\hline ADXEC.28165.C1_at & ANGPT1 & ADXECADA.3575_at & LRP8 \\
\hline ADXEC.11758.C1_at & ANKRD29 & ADXEC.15165.C1_at & LRP8 \\
\hline ADXECAD.11878_s_at & ANKRD29 & ADXEC.8514.C1_x_at & METTL7A \\
\hline ADXPCEC.11748.C1_at & ANKRD29 & ADXECADA.24486_at & METTL7A \\
\hline ADXPCEC.11748.C1_x_at & ANKRD29 & ADXECNTDJ.5826_s_at & METTL7A \\
\hline ADXPCEC.865.C1_at & ANKRD29 & ADXECADA.16813_at & METTL7A \\
\hline ADXPCEC.865.C1_x_at & ANKRD29 & & \\
\hline ADXEC.4289.C1_at & TOM1L2 & & \\
\hline ADXEC.32336.C1_at & NAMPT & ADXEC.11904.C1_at & SLIT3 \\
\hline ADXECADA.10815_at & NAMPT & ADXEC.19191.C1_at & SLIT3 \\
\hline ADXEC.2660.C1-a_s_at & NAMPT & ADXEC.19191.C1_x_at & SLIT3 \\
\hline ADXEC.2660.C1_at & NAMPT & ADXEC.4613.C1_at & SLIT3 \\
\hline ADXECAD.1453_s_at & NAMPT & ADXECAD.25829_s_at & SLIT3 \\
\hline ADXECAD.24382_s_at & NAMPT & ADXECADA.16195_x_at & SLIT3 \\
\hline ADXEC.10262.C1_at & PCM1 & ADXECAD.5047_at & SPP1 \\
\hline
\end{tabular}




\begin{tabular}{|l|l|l|l|}
\hline ADXEC.10262.C1_s_at & PCM1 & ADXECAD.23641_s_at & SPP1 \\
\hline ADXEC.23999.C1_s_at & PCM1 & ADXOCEC.14560.C1_at & SPP1 \\
\hline ADXEC.10262.C2_s_at & PCM1 & ADXOCEC.14560.C1_x_at & SPP1 \\
\hline ADXEC.25350.C1_at & PCM1 & ADXEC.26864.C1_at & TNS1 \\
\hline ADXEC.4679.C2-a_s_at & PLAU & ADXECAD.24662_at & TNS1 \\
\hline ADXECAD.24282_at & PLAU & ADXECEMUTR.2541_s_at & TNS1 \\
\hline ADXEC.21964.C2_at & PLCB4 & ADXEC.3298.C1_at & TNS1 \\
\hline ADXECAD.27780_at & PLCB4 & ADXECADA.10891_at & TOM1L2 \\
\hline ADXECAD.27780_s_at & PLCB4 & ADXEC.13429.C1_at & TOM1L2 \\
\hline ADXECRS.4084_s_at & PLCB4 & ADXEC.4289.C1_at & TOM1L2 \\
\hline ADXEC.21189.C1_s_at & PTGES & ADXEC.21443.C1_at & TSPAN5 \\
\hline ADXECADA.20482_at & PTGES & ADXEC.34129.C1_at & TSPAN5 \\
\hline ADXECADA.20482_x_at & PTGES & ADXECAD.10916_at & TSPAN5 \\
\hline ADXEC.10125.C1_at & RECK & ADXECAD.13537_at & TSPAN5 \\
\hline ADXEC.10125.C1_s_at & RECK & ADXEC.15643.C1_at & TSPAN5 \\
\hline ADXECAD.2455_s_at & RECK & ADXEC.8833.C1_s_at & TSPAN5 \\
\hline ADXECAD.11511_at & RECK & ADXEC.4902.C1_at & TTYH3 \\
\hline ADXEC.25901.C1_at & RGCC & ADXEC.4902.C1_x_at & TTYH3 \\
\hline ADXEC.10181.C1_s_at & RGCC & ADXEC.4902.C2_s_at & TTYH3 \\
\hline ADXEC.3228.C1_s_at & RRM2 & ADXEC.25748.C1_at & VCAN \\
\hline ADXECRS.44245_at & RRM2 & ADXEC.4587.C1-a_s_at & VCAN \\
\hline ADXECNTDJ.6564_at & SEC14L4 & ADXEC.4587.C1_s_at & VCAN \\
\hline ADXECEMUTR.6644_at & SERPINH1 & ADXECAD.17188_at & VCAN \\
\hline ADXECNTDJ.6701_s_at & SERPINH1 & ADXECAD.17188_s_at & VCAN \\
\hline ADXEC.1556.C1_at & SFN & ADXECAD.21682_s_at & VCAN \\
\hline ADXEC.13004.C1_at & SLC27A5 & ADXECEMUTR.4185_s_at & VCAN \\
\hline ADXEC.13004.C1_s_at & SLC27A5 & ADXECNTDJ.1389_at & VCAN \\
\hline ADXEC.13004.C1_x_at & SLC27A5 & ADXECRS.7864_s_at & VCAN \\
\hline ADXECADA.15636_at & SLC27A5 & ADXECADA.19309_at & VSIG10 \\
\hline ADXECNTDJ.6726_at & SLC27A5 & & \\
\hline & & & \\
\hline
\end{tabular}


Supplementary Table 4. The list of Agilent Human $4 \times 44 k$ v2 array probes used in the analysis and the genes to which they map

\begin{tabular}{|l|l|l|l|}
\hline Probe ID & Gene & Probe ID & Gene \\
\hline A_23_P118615 & ABCA8 & A_33_P3364864 & NAMPT \\
\hline A_33_P3342305 & ABCA8 & A_33_P3364869 & NAMPT \\
\hline A_23_P218858 & ABI3BP & A_33_P3375934 & NAMPT \\
\hline A_33_P3411975 & ABI3BP & A_23_P82950 & PCM1 \\
\hline A_33_P3411980 & ABIBB & A_24_P555510 & PCM1 \\
\hline A_23_P350512 & ADAM12 & A_23_P24104 & PLAU \\
\hline A_33_P3256848 & ADAM12 & A_33_P3306146 & PLAU \\
\hline A_33_P3310929 & ADAM12 & A_33_P3260634 & PLCB4 \\
\hline A_33_P3310189 & ADRB1 & A_24_P403417 & PTGES \\
\hline A_24_P152968 & AKR1C1 & A_23_P83028 & RECK \\
\hline A_23_P216023 & ANGPT1 & A_33_P3269899 & RECK \\
\hline A_23_P412577 & ANKRD29 & A_24_P225616 & RRM2 \\
\hline A_23_P209347 & ANKRD44 & A_33_P3389286 & SFN \\
\hline A_24_P128713 & ANKRD44 & A_23_P4611 & SLC27A5 \\
\hline A_23_P212050 & BCHE & A_23_P58588 & SLIT3 \\
\hline A_23_P26024 & C15orf48 & A_33_P3391496 & SLIT3 \\
\hline A_24_P935491 & COL3A1 & A_23_P7313 & SPP1 \\
\hline A_23_P83818 & COL5A1 & A_24_P105733 & TNS1 \\
\hline A_33_P3383351 & COL5A1 & A_33_P3209491 & TNS1 \\
\hline A_33_P3256952 & EGLN3 & A_33_P3738458 & TNS1 \\
\hline A_23_P200222 & LRP8 & A_23_P141180 & TOM1L2 \\
\hline A_23_P415021 & METTL7A & A_33_P3325349 & TSPAN5 \\
\hline & & A_23_P331895 & TTYH3 \\
\hline & & A_23_P144959 & VCAN \\
\hline
\end{tabular}

